\title{
Advance on synthesis, pharmacokinetics, and pharmacodynamics of the anti-epileptic drug: Levetiracetam
}

\author{
Wei Wang \\ Department of Chemistry, Dickinson College, Carlisle, PA, USA
}

\begin{abstract}
Over the past few decades, many patients around the world have suffered from epilepsy, and epilepsy treatment is a long-term process. Thus, the safety and efficacy of medication for epilepsy are of equal importance. Levetiracetam is a second-generation anti-epileptic drug with a unique mechanism of action, good anti-epileptic efficacy, and safety. The therapeutic field has broad application prospects because levetiracetam offers several advantages over other anti-epileptic drugs. In this review, the synthesis, pharmacokinetics, pharmacodynamics, and reverse reaction of levetiracetam were summarized and discussed. This paper will be helpful for the development and application of levetiracetam.
\end{abstract}

\section{Introduction}

Epilepsy is a chronic neurological disorder resulting from excessive and abnormal neuronal activity in the cortex of the brain. It is characterized by sudden recurrent episodes of sensory disturbance, loss of consciousness, muscle spasms, or convulsions. ${ }^{1}$ The seizures of epilepsy have many possible causes, and there are several types of seizures. An abnormality in brain wiring, an imbalance of neurotransmitters, changes in critical features of the channel, or a combination of those factors can trigger epilepsy. It can occur as a result of acquired brain injury or a genetic disorder such as a trauma or stroke, but most of the causes are unknown. ${ }^{2} \mathrm{~A}$ person is considered to have epilepsy only when he has had two or more seizures or an epilepsy syndrome. ${ }^{3}$ It was reported that about 65 million people worldwide suffered from epilepsy, and nearly $80 \%$ of the patients were from developing countries. Epilepsy-related deaths increased from 112,000 in 1990 to 125,000 in $2015 .{ }^{4}$ It is common for people with epilepsy to develop behavioral and emotional problems, especially in conjunctions with seizures.

Epilepsy may be life-threatening because many people with epilepsy are at particular risk of abnormally prolonged seizure and unexplained death. ${ }^{5}$ Epilepsies cannot be cured, but up to $70 \%$ of cases are controllable with medication, diet, devices, or surgery. ${ }^{6,7}$ Most seizures do not damage the brain, but ongoing uncontrolled seizures may cause the brain damage. Thus, epilepsy may cause the premature death and lost work efficiency, and responded to $0.5 \%$ of the global burden of disease. ${ }^{8}$ In this context, the effectiveness, lack of incidence of the adverse reaction, be deficient in drug interconversion, and tolerance can afford substantial benefits for patients with epilepsy and health-care budgets. The research of Anti-epileptic drugs (AEDs) have undergone more than 100 years of development, from the initial application of potassium bromide to the appearance of Phenobarbital in 1912, and to the 1990s, traditional AEDs such as Phenytoin, Primidone, Ethosuximide, Valproic acid, and Carbamazepine have come out one after another. ${ }^{9}$ The significant progress has been made in the treatment of epilepsy. However, after a reasonable single or combined treatment of traditional AEDs, drug tolerance, which is refractory epilepsy, is still present in $20 \%$ to $30 \%$ of patients. ${ }^{10,11}$ After the 1990 s, researches are mainly focused on refractory epilepsy and developed and marketed a new generation of AEDs, including Felbamate, Gabapentin, Lamotrigine, levetiracetam, Oxcarbazepine, Tiagabine, Topiramate, Aminocaproic acid, and Zonisamide. ${ }^{11,12}$ The new generation of AEDs has the advantages of better antiepileptic efficacy, lower incidence of adverse reactions, better tolerance, fewer drug interactions, and better pharmacokinetic properties. ${ }^{9,10,11,12,13}$ Among secondgeneration AEDs, Levetiracetam was an acetylcholine agonist developed by UCB of Belgium and related to piracetam. ${ }^{14,15}$ It was mainly used to treat localizationrelated epilepsies, secondary epilepsy, and generalized epilepsy, approved by the FDA in April 2000. ${ }^{15,16}$

Levetiracetam is the $(\mathrm{S})$-enantiomer of the racemic compound etiracetam, its chemical name (S)-2-(2oxopyrrolidin-1-yl) butanamide and product name is Keppra. ${ }^{14}$ It is a pyrrolidone derivative with empirical formula $\mathrm{C}_{8} \mathrm{H}_{14} \mathrm{~N}_{2} \mathrm{O}$, and has a low molecular weight 170 . $209 \mathrm{~g} / \mathrm{mol}$. ${ }^{14,17}$ The molecular structure is shown in Figure 1. The Levetiracetam is a crystalline powder with a white to off-white color and a faint odor and bitter taste. ${ }^{14,18}$ Levetiracetam has high solubility in aqueous solutions with a melting point of $117^{\circ} \mathrm{C}$, a topological surface area of $63.4 \AA$, and an $\mathrm{xLogP}$ of $-0.62 . .^{18,19}$ Moreover, the levetiracetam molecule contains four hydrogen bond donors, one hydrogen bond acceptors,

"email: oneweiway@gmail.com 
three rotatable bonds, and compiling with Lipinski's rules.<smiles>CC[C@@H](C(N)=O)N1CCCC1=O</smiles>

Figure. 1. Chemical structure of levetiracetam.

Levetiracetam exhibits strong anti-epileptic properties and can effectively control the onset of epilepsy. The drug not only has a high therapeutic index but also has unique pharmacokinetic characteristics. After oral ingestion, levetiracetam is rapidly absorbed in the small intestine, and its bioavailability is approximately $100 \% .{ }^{17}$ The pharmacokinetic distribution is close to the ideal kinetic distribution model of antiepileptic drugs. Besides, Levetiracetam is a broadspectrum anti-epileptic drug with unique structure and mechanism of action. Compared to other AEDs, it is extremely safe to be used as an add-on or monotherapy treatment for partial, and generalized tonic-clinic seizures. ${ }^{9,12,13}$

In this article, the structure-activity relationship and synthesis of levetiracetam were discussed. Its outstanding pharmacokinetic and pharmacological profile and reverse reactions were also discussed.

\section{Synthesis}

Several chemical synthesis pathways of levetiracetam have been reported in previous studies. The conventional chiral resolution generally synthesizes the racemic form of alpha-ethyl-2-oxo-1-pyrrolidine acetamide and then dissolves (ammonized) to obtain levetiracetam. Jean $\mathrm{G}$ et al. reported that the starting material 2-Pyrrolidinone could react with sodium methoxide to form the sodium salt of 2-pyrrolidone (Figure 2). ${ }^{\mathbf{2 0}}$ The racemic mixture passed through a resolving agent (R)- $(+)-\alpha-\mathrm{N}-$ Methylbenzylamine and was resolved to give a monomeric acid, which could be ammoniated to give the target product levetiracetam. ${ }^{16,21}$ The advantage of this route was that it could eliminate the cyclization step of levetiracetam. However, the main disadvantages were hat the loss amount in the enantiomeric resolution process was large, the yield was meager, the resolving reagent was expensive, and the process route was complicated. The (R)-enantiomer had only a slight or insignificant effect on the inhibition of seizures, while levetiracetam was a safe and effective anti-epileptic drug. ${ }^{21}$ As a result, the preparation of pure levetiracetam needed a more effective method.

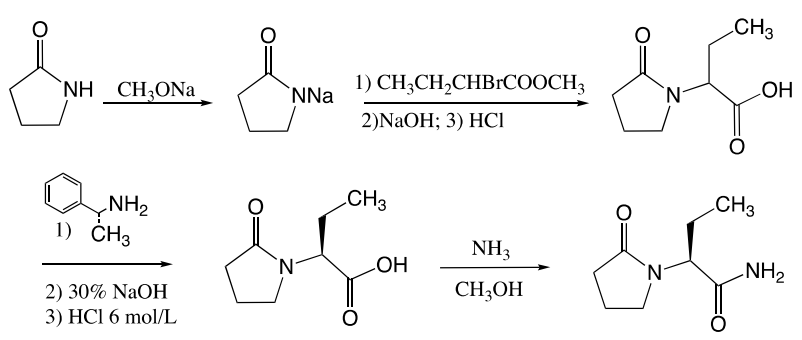

Figure. 2. The first synthesis of levetiracetam by Gobert from 1990.

Levetiracetam can also be synthesized with LMethionine as starting material. As Figure 3 shown, the preparation route was carried out by a series of reaction of desulfurization, methylation, esterification, ammonification, amidation, and intramolecular ringclosure to give a yield around $44.6 \%{ }^{.22}$ In this route, the amount of nickel used during desulfurization was tenfold more greater than that of L-Methionine, which cannot be reused and its production cost was high. For this reason, Shen incorporated improvement over this reaction with the same starting material L-Methionine (Figure 4). ${ }^{23}$ Without the usage of nickel, idomethane was reacted with alkaline reagent such as sodium carbonate and potassium carbonate to produce LHomoserine, which was able to react with 4chlorobutyryl chloride and methanesulfonyl chloride under alkaline condition. The acylamide generated via ammonification can further cyclize under alkaline condition, and finally reduced to Levetiracetam. Although the method of Shen avoids high cost of nickel, it involved various side reactions during the reaction of L-Homoserine and 4-chlorobutyryl chloride, making resultant hard to isolate and thus giving a low reaction yield.

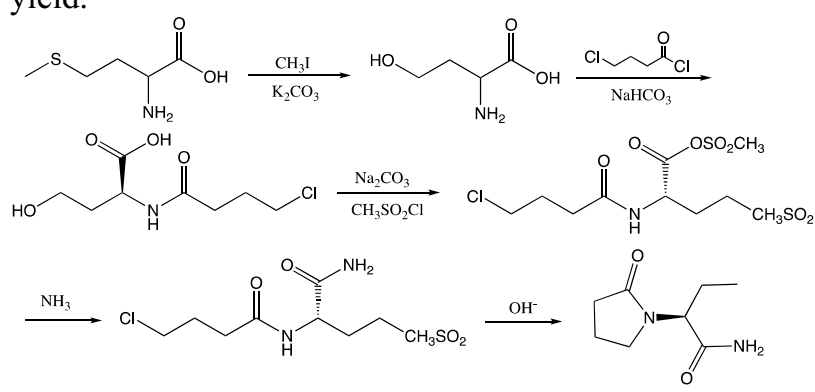

Figure. 3. The modified synthesis method of levetiracetam with L-methionine as starting material.<smiles>CCC(C(=O)OC)N1CCCC1=O</smiles>

Figure. 4. The synthesis of levetiracetam with L-methionine as starting material.

Levetiracetam can use (S)-2-Aminobutyric acid as starting material too. The preparation route (Figure 5) was carried out by reacting (S)-2-aminobutyric acid with thionyl chloride to form an acyl chloride, followed by 
esterification with methanol (or ethanol) to obtain an amino ester. Intermolecular nucleophilic substitution of amino ester and ethyl 4-bromobutyrate gave (S)-4-[1(methoxycarbonyl) propyl-amino] butyric acid ethyl ester. The purified intermediate was isolated, and 2-(2Oxo-1-pyrrolidinyl) methyl acetate was obtained by ring closure in the presence of 2-hydroxypyridine. The ester was directly converted to the acylamide by the aminolysis reaction to prepare levetiracetam. ${ }^{24}$ The starting material of this synthetic route was the levorotatory isomer. The separation process of enantiomers was not involved in the whole route. It was only necessary to control the racemic side reaction during the reaction to increase the enantiomer of the product purity. The total synthetic yield of this route can reach about $40 \%$. This process was easy to operate and did not require expensive catalysts and harsh operating conditions. Therefore, it became the primary method of industrial production.
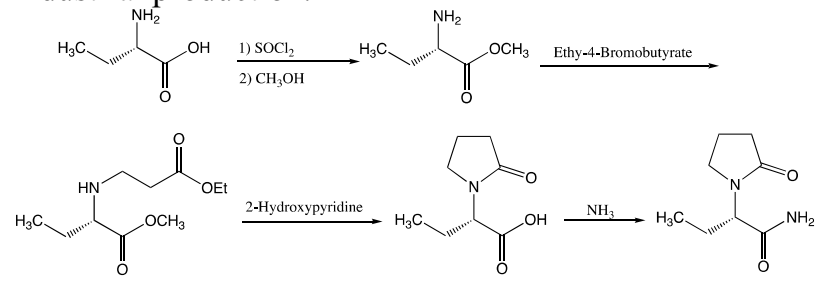

Figure. 5. The synthesis of levetiracetam starting from chiral benzyl glycidyl ether. ${ }^{24}$

Francesca etc. developed a novel route that started from (S)-2-Bromobutyric acid, an organobromine compound, to synthesize levetiracetam and its enantiomer (Figure 6). ${ }^{\mathbf{2 5}}$ With the presence of N, N'Dicyclohexylcarbodiimide and control of temperature below $-78^{\circ} \mathrm{C}$, an ester intermediate formed. Followed by the decrease of lithium hydroxide, S-2-Bromobutyric acid was formed and further reacted with 2pyrrolidinone to produce (2-Oxopyrrolidin-1-yl)acetic acid, which can result in the synthesis of levetiracetam via esterification and ammonolysis. In this route, the stringent requirement of low temperature was the main factor that dominates the reaction. As Figure 7 shown, the route was based on butyraldehyde using nitrosobenzene and L-proline as auxiliaries, requiring temperature under $20{ }^{\circ} \mathrm{C}$ and $20 \mathrm{~h}$ reaction time. The resultant became chiral intermediate through reduction of sodium borohydride and further reduced to (R)-1,2Butanediol by $\mathrm{Pd} / \mathrm{C}$. By blocking the 1-hydroxy group by dibutyltin oxide and inactivating another hydroxy group by methanesulfonyl chloride under room temperature, the resultant mixture can react with 2pyrrolidinone to produce (S)-2-(2-Oxopyrrolidin-1-yl) butanoic acid, in which the butyl group can be hydrolyzed. Then its hydrolysis product reacted with ethyl chloroformate and triethylamine, and through ammonolysis and recrystallization by acetone to produce levetiracetam. ${ }^{26}$ The process had a complex operation, required utilization of expensive organic tin compounds, and has a low yield of $33 \%$.

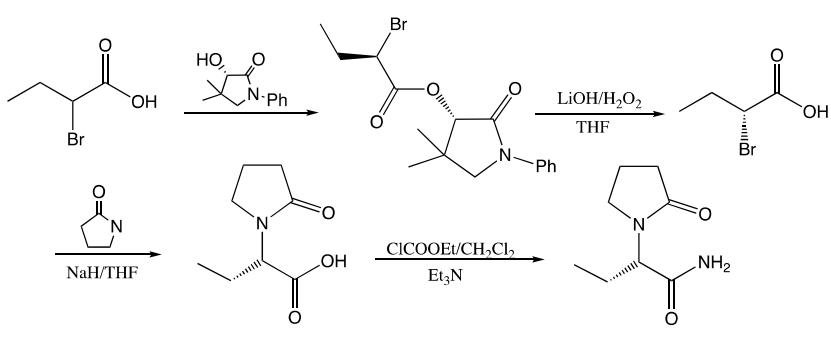

Figure. 6. the synthesis of levetiracetam from (s)-2bromobutyric acid.

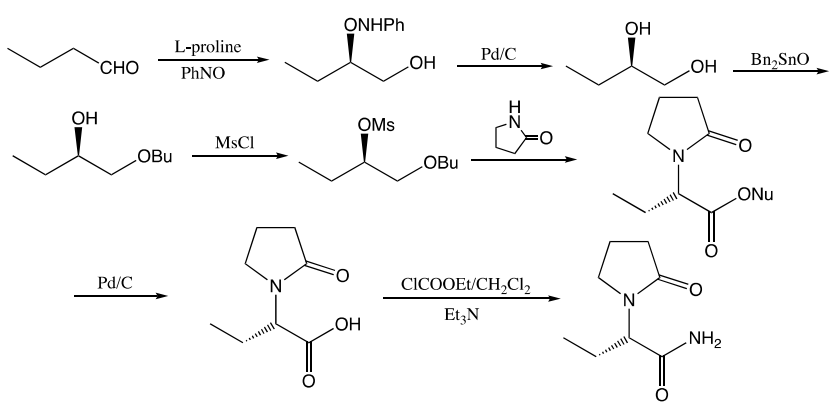

Figure. 7. The synthesis of levetiracetam from butyraldehyde.

For the synthesis of asymmetric catalytic hydrogenation carried out with intermediate containing alkenes, metal complexes are commonly used to catalyze its alkenes' carbon-carbon bonds to obtain levetiracetam with high-selectivity. As Figure 8 shown, the synthesis adopted rhodium $(\mathrm{Rh})$ or ruthenium $(\mathrm{Ru})$ chelate as a chiral catalyst. ${ }^{27}$ Firstly, methyl 2-oxobutanoate reacted with 2-pyrrolidinone in the presence of catalyst (the amount of catalyst was $0.5-2.5 \%$ of the substrate) to produce (Z)-2-(2-oxotetrahydro-1H-1-pyrrolyl)-2butenoic acid. After ammonolysis, in the pressurized hydrogen atmosphere, the resultant (Z)-2-(2oxotetrahydro-lH-l-pyrrolyl)-2-butenenamide was catalyzed and hydrogenated to obtain (S)-(-)- $\alpha$-ethyl-2oxo- 1-pyrrolidine acetamide through separation and purification with the yield around $83 \%$. For synthesis routes, in which alkene intermediate adopted rhodium chelate as a chiral catalyst to preform asymmetric catalytic hydrogenation, reaction time varied with catalyst types and amount of usage. Although this route was relatively direct and straightforward solvent distillation can isolate enantiomer with high-selectivity and yield over $85 \%$, rare mental chelate as its chiral catalyst was required and difficult to recycle, making it became a costly choice for industrial production.

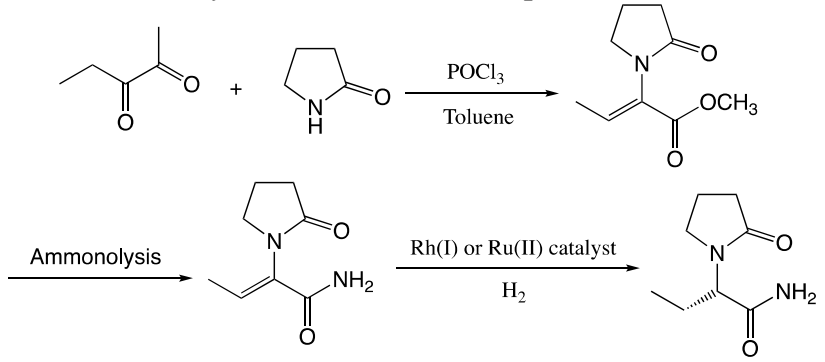

Figure 8. Synthesis of levetiracetam by asymmetric catalytic hydrogenation. 


\section{Pharmcokintics}

The pharmacokinetics of anti-epileptic drugs were of concern because epilepsy was a disease that required long-term medication, which also required the monitoring of pharmacokinetics; many patients used two or more anti-epileptic drugs. Therefore, a reasonable dosage regimen was required, and the interaction between drugs was also significant; besides, patients with epilepsy can also receive medication for other diseases, which also increased the possibility of interconversion with non-epileptic drugs and toxicity. In order to have a good curative effect, ideal AEDs should have bioavailability close to $100 \%$, fast absorption, linear pharmacokinetics, the long half-life, lack of plasma protein binding, and no drug interconversions.

Levetiracetam had almost all the metabolic kinetic properties of an ideal anti-epileptic drug. Follow by oral administration, levetiracetam was a highly soluble compound with an absolute bioavailability of nearly $100 \%$ and an absorption rate greater than $95 \%{ }^{28}$ The maximum plasma concentration can be reached within 0.6 to 1.3 hours and can achieved steady-state concentration within two days by taking twice a day. ${ }^{29}$ Although oral administration with food tended to delay $t_{\max }$ to $1.5 \mathrm{~h}$ and lower $C_{\max }$ by $20-30 \%$, there was little effect on the extent of absorption. ${ }^{28}$ In the different dose studies, a linear, predictable steady-state pharmacokinetics was shown. ${ }^{16}$ The average elimination half-life was about 7 hours, so that it can be administered twice a day. Single or multiple administrations of 500 mg tablets did not affect absorption or absorption. Moreover, levetiracetam and its primary metabolites were difficult to bind to plasma proteins $(<10 \%))^{30}$ as a result, little drug interconversion through competition for protein binding was likely with levetiracetam. In-vivo tests had confirmed that its pharmacokinetics were not affected by other anti-epileptic drugs (AEDs) that were often used in combination, and would ot affect plasma concentrations of other AEDs such as Phenytoin, Carbamazepine, Valproic acid, Phenobarbital, and Primidone, or pharmacokinetics of the oral contraceptives (Ethinylestradiol, Levonorgestrel), Digoxin, and Warfarin. ${ }^{30}$ The apparent volume of distribution $\left(\mathrm{V}_{\mathrm{d}}\right)$ was 0.5 to $0.7 \mathrm{~L} / \mathrm{kg}$ for children and 0.7 to $0.9 \mathrm{~L} / \mathrm{kg}$ for adults, which means it was easily dialyzable.

\section{Pharmcodynamics}

Levetiracetam had a novel anti-epileptic mechanism and it was used as a broad-spectrum anti-epileptic agent in both children and adults for adjunct treatment or monotherapy. ${ }^{31}$ However, the action mechanism of levetiracetam action was not well understood in detailed. It was different structurally and mechanistically from any other anti-epileptic class. The pharmacological function of levetiracetam was studied and various mechanisms were described by many scientists in past few years, but none of those work shared similar modes of action related to other anti-epileptic drugs. The possible mechanism might be levetiracetam did not affect GABAergic neurotransmission, lack of binding ability to GABA and glutamate receptors, and had little effect on voltage-dependent sodium channels. ${ }^{22,}$ 32, 33, 34, Levetiracetam could combine with the synaptic vesicle glycoprotein (SV2A) of the central nervous system, which could regulate the extracellular secretion of synaptic vesicles and the release of presynaptic neurotransmitters. Thus, Levetiracetam can lead to modulation of synaptic neurotransmitter release associated with SV2A as a possible mechanism of action. The inhibition of high voltage regulated calcium channels can be a potential mechanism. ${ }^{35},{ }^{36}$ Levetiracetam could eliminate the inhibitory effects of negative allosteric inhibitors ( $\beta$-carbolines and zinc) on GABA and Glycine (Gly) without directly binding to the receptors. ${ }^{22,}{ }^{37}$ The study of the pharmacological actions of levetiracetam was mainly based on animal models of epilepsy. In the acute maximal electroshock epilepsy model and the pentylenetetrazol-induced acute epilepsy model, Levetiracetam had a little anti-epileptic effect but can reduce hippocampal neuronal death in the temporal lobe epilepsy model. ${ }^{38}$ In vivo animal experiments, Levetiracetam showed a significant anti-epileptic effect on kindle and absence seizures by reducing the discharge time after kindle. ${ }^{38,39}$ However, the translational value of observational experiments based on such animal models was too limited to understand human epilepsy and access treatment.

During the monitoring of the long-term treatment, it was found that levetiracetam had no drug resistance in the tested animals, and repeated use after the suspension of the organism did not reduce its drug reactivity. The pharmacological effects of levetiracetam were effective through the drug acting on the central nervous system. In the test, the toxic and side effects of the animal model were still very low in spite of the large number of medications. These results showed that levetiracetam had almost no toxic side effects. As a clinical drug for the treatment of localized and generalized seizures, levetiracetam had a broad spectrum, high efficiency, no drug resistance and toxic side effects. It was fully applicable to the treatment of acquired epilepsy, and has excellent medicinal value from the perspective of medicinal use to prevent the disease.

\section{Adverse effects}

The pharmacokinetics of anti-epileptic drugs were of Levetiracetam was highly safe, well-tolerated, and rare adverse reactions. Central nervous system reactions were common, such as fatigue, dizziness, drowsiness, headache, and children might have signs of excitement and irritability. The slow increase might be avoided. Cereghino and other research results showed that the incidence of adverse reactions was $10 \%$; most of them were mild to moderate (fatigue, drowsiness, headache, dizziness, flu-like symptoms, etc.), which could be tolerated by newborns, infants, and older adults. ${ }^{40}$ Drowsiness was the most common adverse reaction, potentially decreasing daytime activities and increasing 
nerve action potential. However, it had no significant effect on the total time and quality of sleep. Besides, adverse reactions mainly occurred within four weeks of the initial treatment, and the incidence and severity of levetiracetam decreased with the extension of treatment time. Levetiracetam had no significant quantitative correlation in the incidence of adverse reactions. There were some reports that levetiracetam could cause mood disorders and even suicidal tendencies. There were different opinions on whether levetiracetam had an effect on cognition and learning. ${ }^{31,40,41}$

\section{Conclusion}

Epilepsy treatment was a long-term process. Thus, the safety and efficacy of drugs were of equal importance. levetiracetam, as a new type of anti-epileptic drug, had shown outstanding pharmacokinetic and pharmacological activity. Unlike other traditional antiepileptics, it had ideally pharmacokinetic character, which made it is easy to absorb orally, had high bioavailability, and required only twice-daily dosing. Besides, it had novel anti-epileptic mechanisms and could be used as a broad-spectrum anti-epileptic agent in both children and adults for adjunct treatment or monotherapy without interconversion with other drugs.

\section{References}

1. Lawal, M., Omobayo, H., \& Lawal, K. Epilepsy: pathophysiology, clinical manifestations and treatment options. British Journal of Neuroscience Nursing, 14(2), 58-72 (2018)

2. GBD 2015 Disease and Injury Incidence and Prevalence Collaborators.Global, regional, and national incidence, prevalence, and years lived with disability for 310 diseases and injuries, 1990-2015: a systematic analysis for the Global Burden of Disease Study 2015. (2016)

3. Falco-Walter, J. J.; Scheffer, I. E.; Fisher, R. S. The New Definition and Classification of Seizures and Epilepsy. Epilepsy Research, 139, 73-79, (2018)

4. Zhang, X., Li, X., Liu, N., Zheng, P., Ma, L., Guo, F., ... \& Yu, J. The Anticonvulsant Effects of Baldrinal on Pilocarpine-Induced convulsion in Adult Male Mice. Molecules, 24(8), 1617, (2019)

5. Stafstrom, C. E.; Carmant, L. Seizures and Epilepsy: An Overview for Neuroscientists. Cold Spring Harbor Perspectives in Medicine, 5(6), (2016)

6. Sheng, J.; Liu, S.; Qin, H.; Li, B.; Zhang, X. DrugResistant Epilepsy and Surgery. Current Neuropharmacology, 16 (1), (2017)

7. Eadie, M. J. Shortcomings in the Current Treatment of Epilepsy. Expert Review of Neurotherapeutics, 12 (12), 1419-1427, (2012)

8. Naghavi, M. Global, Regional, and National Burden of Suicide Mortality 1990 to 2016: Systematic Analysis for the Global Burden of Disease Study 2016. Bmj, 194, (2019)
9. Anam, S.; Hunter, S.; Afzal, K. The Effects of Antiepileptic Drugs on Pediatric Cognition, Mood, and Behavior. Journal of Pediatric Epilepsy, 06 (01), 003-018, (2016)

10. Dalic, L.; Cook, M. Managing Drug-Resistant Epilepsy: Challenges and Solutions. Neuropsychiatric Disease and Treatment, 12, 2605-2616, (2016)

11. Jacob,S.; Nair A. B. An Updated Overview on Therapeutic Drug Monitoring of Recent Antiepileptic Drugs. Drugs R\&D, 16, 303-316, (2016)

12. French, J, A. New generation antiepileptic drugs: what do they offer in terms of improved tolerability and safety? Therapeutic Advances in Drug Safety, 2(4), 141-158, (2011)

13. Emilio, P., and Svein, J. I. The ideal pharmacokinetic properties of an anti-epileptic drug: how close does levetiracetam come? Disord, 5, 1726, (2003)

14. Keppra XR [Package insert]. Smyrna, GA: UCB Pharma, Inc, (2008)

15. Song, L., Shemchuk, O., Robeyns, K., Braga, D., Grepioni, F., and Leyssens, T. Ionic Cocrystals of Etiracetam and Levetiracetam: The Importance of Chirality for Ionic Cocrystals. Crystal Growth \& Design, 19, 2446-2454, (2019)

16. Brittain, H. G. Profiles of drug substances, excipients, and related methodology. Academic Press, imprint of ElsevierCambridge, MA, (2018)

17. Grace, A. C., Prabha, T., Jagadeeswaran, M., Srinivasan, K., and Sivakumar, T. Simultaneous Estimation of Levetiracetam and its Preservatives In Oral Liquid Dosage form by Rp-Hplc Method. SOJ Materials Science \& Engineering, 4, 1-5, (2016)

18. M.J. O’Neil. The Merck Index-An Encyclopedia of Chemicals, Drugs, and Biologicals, Whitehouse Station,13 p. 978, (2001)

19. Sivaneswari, S., Karthikeyan, E., Veena, D., Chandana, P. J., Sai, P. S., Subhashree, P., Ramya, L., Rajalakshmi, R., and Ashok, C. K. K. Physiochemical characterization of taste masking levetiracetam ion exchange resinates in the solid state and formulation of stable liquid suspension for pediatric use. Beni-Suef University Journal of Basic and Applied Sciences, 5, 126-133, (2016)

20. J. Gobert, J.-P. Geerts, G. Bodson. (S)- $\alpha$-ethyl-2oxo-1-pyrrolidineacetamide Compositions, US Patent 4943639A, (1985)

21. Gower, A. J., Noyer, M., Verloes, R., Gobert, J., and Wülfert, E. ucb L059, a novel anti-convulsant drug: pharmacological profile in animals. European Journal of Pharmacology 230, 389, (1993)

22. Zhou, X., Xu, G., Zhou, Y., Xu, M. Synthesis of Anticonvulsant Drug Levetiracetam[J]. Fine Chemical Intermediates, 35(3), 27-28, (2005) 
23. Shen, L., Bian, Y., Liu, F., Wu, P., Jiang, K. A method for preparing levetiracetam[P]. 200910026351.9, (2009)

24. Ates C, Surtees J, Burteau A-C, et al. Oxopyrrolidine compounds, preparation of said compounds and their use in the manufacturing of levetiracetam and analogues.US Patent WO03014080, (2003)

25. Boschi F, Camps P, Comes-Franchini M. A synthesis of levetiracetam based on (S)-Nphenylpantolactam as a chiral auxiliary[J]. Tetrahedron: Asymmetry, 16(22): 3739-3745, (2005)

26. Kotkar S P, Sudalai A. A short enantioselective synthesis of the antiepileptic agent, levetiracetam based on proline-catalyzed asymmetric $\alpha$ aminooxylation $[\mathrm{J}]$. Tetrahedron Letters. 47(38): 6813-6815, (2006)

27. Surtees J, Marmon V, Differding E. 2-Oxo-1pyrrolidine derivatives, process for preparing them and their uses: US6,713,635[P], (2004)

28. Browne, T. R. Pharmacokinetics of anti-epileptic drugs. Neurology, 51(5 Suppl 4), 2-7, (1998)

29. Wright, C., Downing, J., Mungall, D., Khan, O., Williams, A., Fonkem, E., Garrett, D., Aceves, J., and Kirmani, B. Clinical Pharmacology and Pharmacokinetics of Levetiracetam. Frontiers in Neurology 4, (2013)

30. Patsalos, P. Pharmacokinetic profile of levetiracetam. Pharmacology \& Therapeutics, 85, 77-85, (2000)

31. Abou-Khalil. B. Levetiracetam in the Treatment of Epilepsy. Neuropsychiatric Disease and Treatment, 507, (2008)

32. Zona, C., Niespodziany, I., Marchetti, C., Klitgaard, H., Bernardi, G., and Margineanu, D. G. Levetiracetam does not modulate neuronal voltagegated $\mathrm{Na}$ and T-type Ca2 currents. Seizure, 10, 279286, (2001)

33. Klitgaard, H. Levetiracetam: The Preclinical Profile of a New Class of Anti-epileptic Drugs Epilepsia 42, 13-18, (2001)

34. Madeja, M., Margineanu, D. G., Gorji, A., Siep, E., Boerrigter, P., Klitgaard, H., and Speckmann, E.-J. Reduction of voltage-operated potassium currents by Levetiracetam: a novel anti-epileptic mechanism of action? Neuropharmacology, 45, 661-671, (2003)

35. Rajakulendran, S., and Hanna, M. G. The Role of Calcium Channels in Epilepsy. Cold Spring Harbor Perspectives in Medicine, 6, (2016)

36. Lukyanetz, E., Shkryl, V., and Kostyuk, P. Selective Blockade of N-Type Calcium Channels by Levetiracetam. Epilepsia, 43, 9-18, (2002)

37. Rigo, J.-M., Hans, G., Nguyen, L., Rocher, V., Belachew, S., Malgrange, B., Leprince, P., Moonen, G., Selak, I., Matagne, A., and Klitgaard, H. The anti-epileptic drug levetiracetam reverses the inhibition by negative allosteric modulators of neuronal GABA- and glycine-gated currents. British Journal of Pharmacology, 136, 659-672, (2002)

38. Sharief, M., Singh, P., Sander, J., Patsalos, P., and Shorvon, S. Efficacy and tolerability study of ucb L059 in patients with refractory epilepsy. Journal of Epilepsy, 9, 106-112, (1996)

39. Klitgaard, H., Matagne, A., Gobert, J., and Wülfert, E. Evidence for a unique profile of Levetiracetam in rodent models of seizures and epilepsy. European Journal of Pharmacology, 353, 191-206, (1998)

40. Cereghino, J. J., Biton, V., Abou-Khalil, B., Dreifuss, F., Gauer, L. J., and Leppik, I. Levetiracetam for partial seizures: Results of a double-blind, randomized clinical trial. Neurology, 55, 236-242, (2000)

41. Brockmoller, J., Thomsen, T., Wittstock, M., Coupez, R., Lochs, H., and Roots, I. Pharmacokinetics of levetiracetam in patients with moderate to severe liver cirrhosis (Child-Pugh classes A, B, and C): Characterization by dynamic liver function tests. Clinical Pharmacology \& Therapeutics , 77, 529-541, (2005) 